\title{
Zero-Disturbance Control of Free-Floating Space Manipulators Using Integral-Type Sliding Mode Control
}

\author{
Heping $\mathrm{Li}^{1}$ and $\operatorname{Ren} \mathrm{Li}^{2}$ \\ ${ }^{1}$ Department of Electronic and Information Engineering, Loudi Vocational \& Technical College, Louxing District, Loudi, \\ Hunan 417000, China \\ ${ }^{2}$ Chongqing SANY High-Intelligent Robots Co., Ltd., Chongqing 401120, China
}

Correspondence should be addressed to Heping Li; lhp13973812001@sina.com

Received 13 July 2014; Accepted 14 September 2014

Academic Editor: Xudong Zhao

Copyright (C) 2015 H. Li and R. Li. This is an open access article distributed under the Creative Commons Attribution License, which permits unrestricted use, distribution, and reproduction in any medium, provided the original work is properly cited.

\begin{abstract}
A free-floating space manipulator is an underactuated system, of which the spacecraft is permitted to rotate freely in response to the manipulator motions. The dynamic coupling property between the spacecraft and the manipulator makes motion control of such systems a significant challenge. In the paper, a zero-disturbance control method for free-floating space manipulators operating in task space is presented. An explicit direct relationship between the spacecraft attitude quaternions and the manipulator joint variables is established using nonholonomic constraints of the angular momentum conservation. By this means the kinematic redundancy of the system is used to adjust the spacecraft attitude. An integral-type sliding mode controller with adaptive switching gains is developed for coordinated motion control of the spacecraft and the manipulator. Simulations on three-link planar model show that the spacecraft remains undisturbed during the whole process of manipulations, which confirms the effectiveness of the proposed method.
\end{abstract}

\section{Introduction}

Space manipulators will play an important role in complex space missions, such as large structure assembling, on-orbit repairing, and capturing $[1,2]$. A space manipulator system consists of a spacecraft and an $\mathrm{N}$-degree-of-freedom robotic manipulator. Generally, in order to increase the system's service span, more importantly to avoid interaction with the target, it is desirable to have the spacecraft control system turned off during manipulating $[3,4]$. Consequently, the spacecraft is permitted to translate or rotate freely in response to the reaction of manipulator's movement. For such a system, it is termed as free-floating space manipulator systems.

A free-floating space manipulator has properties of strong nonlinearities, nonholonomic constraints, and dynamic singularities $[5,6]$. Therefore, control of free-floating space manipulators becomes a challenging task [7, 8]. Dubowsky and Papadopoulos [9] investigated the kinematics, dynamics, and control of space manipulator systems. $\mathrm{Xu}$ et al. [10] and Papadopoulos et al. [11] developed a polynomial function based planning method for simultaneous control of spacecraft attitude and manipulator using manipulator actuators only. Recently Xu et al. [12] proposed coordinated trajectory planning methods to stabilize the base attitude and the centroid position at the same time. Yoshida [13] introduced a reaction null-space (RNS) concept in order to plan zero-disturbance end-effector paths. In [14] the RNS is applied to reactionless motion control of free-floating space manipulators. Liao et al. [15] have studied translational zerodisturbance curve and its application to zero-disturbance motion planning. In addition, many articles [16-21] proposed kinds of control methods for space manipulators aimed at maintaining the base orientation while manipulating. However, most of them require using reaction wheels or reaction jets to stabilize the base. Researches on coordinated control of both spacecraft attitude and manipulator motions, utilizing dynamic coupling of the free-floating systems, are not enough [8].

The focus of this paper is on controlling manipulator joints to track trajectories in task space, meanwhile retaining 
the spacecraft attitude undisturbed. A control method based on integral-type sliding manifold is proposed. After that, the adaptive reaching law is designed to improve the control performances. The paper is organized as follows. In Section 2, problem formulation of free-floating space manipulators is addressed. In Section 3, the proposed zero-disturbance control method is presented in detail. In Section 4, simulations are conducted to demonstrate the effectiveness of the proposed method. Finally, Section 5 concludes the paper.

\section{Problem Formulation}

2.1. Dynamics and Kinematics Equations. Given a freefloating space manipulator system, the control system of the spacecraft is closed during manipulating. Assuming that there are no external forces and torques acting on it, the linear and angular momentums remain constant. Under further assumption of initial rest, the system center of mass (CM) remains fixed in inertial frame. Therefore, the origin of inertial frame can be chosen to be the system's CM.

The end-effector linear velocity vector is given by

$$
\dot{\mathbf{r}}_{\mathrm{end}}=\mathrm{J}_{L}(\lambda, \mathbf{q}) \cdot \dot{\mathbf{q}},
$$

where vectors $\mathbf{q}, \dot{\mathbf{q}} \in \mathfrak{R}^{N}$ represent the manipulator joint angles and rates, respectively; $\mathbf{J}_{L}(\boldsymbol{\lambda}, \mathbf{q}) \in \mathfrak{R}^{3 \times N}$ is submatrix of the generalized Jacobian matrix relative to linear velocity, expressed as a function of $\boldsymbol{\lambda}$ and $\mathbf{q}$ with $\boldsymbol{\lambda}$ being the spacecraft attitude defined in the following text.

Considering the preceding conditions, the equations of motion of free-floating space manipulators can be written as follows, in which the nonholonomic constraints are included:

$$
\mathbf{H}(\mathbf{q}) \ddot{\mathbf{q}}+\mathbf{C}(\mathbf{q}, \dot{\mathbf{q}})=\boldsymbol{\tau},
$$

where $\mathbf{H}(\mathbf{q}) \in \mathfrak{R}^{N \times N}$ is a positive definite symmetric matrix and $\boldsymbol{\tau}$ is the joint torque vector, with $\tau=\left[\tau_{1}, \tau_{2}, \ldots, \tau_{N}\right]^{T}$. $\mathbf{C}(\mathbf{q}, \dot{\mathbf{q}})=\left[\dot{\mathbf{H}}(\mathbf{q})-(1 / 2) \dot{\mathbf{q}}^{T}(\partial \mathbf{H}(\mathbf{q}) / \partial \mathbf{q})\right] \cdot \dot{\mathbf{q}} \in \mathfrak{R}^{N}$ contains the nonlinear Coriolis and centrifugal terms. The detailed expression of $\mathbf{H}(\mathbf{q})$ and $\mathbf{C}(\mathbf{q}, \dot{\mathbf{q}})$ can be found in [3].

\subsection{Spacecraft Attitude Kinematics with Nonholonomic Con-} straints. For a free-floating system, the conservation of angular momentum is unintegrable. Therefore, the nonholonomic constraints between the spacecraft angular velocity and the manipulator joint rates can be written as

$$
\omega_{0}=\Gamma \cdot \dot{\mathbf{q}}
$$

where $\boldsymbol{\omega}_{0}$ is the spacecraft angular velocity, $\boldsymbol{\Gamma}=$ $-\mathbf{D}^{-1}(\lambda, \mathbf{q}) \mathbf{D}_{q}(\lambda, \mathbf{q})$ with $\mathbf{D}(\lambda, \mathbf{q}) \in \mathfrak{R}^{3 \times 3}$ and $\mathbf{D}_{q}(\lambda, \mathbf{q}) \in$ $\mathfrak{R}^{3 \times N}$ being the inertia-type matrices, and $\mathbf{D}(\lambda, \mathbf{q})$ is always invertible.

As mentioned above, $\boldsymbol{\lambda}$ adopted to describe the spacecraft attitude is the unit quaternion. The attitude kinematics in terms of unit quaternion with respect to inertial frame is given by

$$
\dot{\lambda}=\left[\begin{array}{c}
\dot{\lambda}_{0} \\
\dot{\bar{\lambda}}
\end{array}\right]=\frac{1}{2}\left[\begin{array}{c}
-\bar{\lambda}^{T} \\
\Lambda^{T}
\end{array}\right] \boldsymbol{\omega}_{0}
$$

where $\boldsymbol{\Lambda}=\lambda_{0} \mathbf{I}+\bar{\lambda}^{\times}, \bar{\lambda}=\left[\begin{array}{lll}\lambda_{1} & \lambda_{2} & \lambda_{3}\end{array}\right]^{T} . \mathbf{I} \in \mathfrak{R}^{3 \times 3}$ is an identity matrix and $\bar{\lambda}^{\times}$is a skew-symmetric matrix. Obviously, $\left[\lambda_{0}, \bar{\lambda}\right]^{T}$ and $\left[-\lambda_{0},-\bar{\lambda}\right]^{T}$ represent identical orientation. In the paper, $\lambda_{0}$ is bounded by $\lambda_{0} \geq 0$.

Furthermore, the second-order derivative of the quaternion is related to angular accelerations by differentiating (4) (see Appendix for details) as follows:

$$
\left[\begin{array}{c}
\ddot{\lambda}_{0} \\
\ddot{\bar{\lambda}}
\end{array}\right]=\frac{1}{2}\left[\begin{array}{c}
-\bar{\lambda}^{T} \\
\boldsymbol{\Lambda}^{T}
\end{array}\right][\dot{\boldsymbol{\Gamma}} \cdot \dot{\mathbf{q}}+\boldsymbol{\Gamma} \cdot \ddot{\mathbf{q}}]-\frac{1}{4} \dot{\mathbf{q}}^{T} \boldsymbol{\Gamma}^{T} \boldsymbol{\Gamma} \dot{\mathbf{q}}\left[\frac{\lambda_{0}}{\bar{\lambda}}\right] .
$$

2.3. Equations of Motion in Task Space. Using the property of dynamic coupling, the spacecraft attitude can be adjusted by controlling of manipulator joints. Our objective is to control the end-effector of the manipulator tracking desired trajectories in task space; meanwhile, during the process of manipulation, the spacecraft attitude remains at the original orientation by controlling only the manipulator joints. Obviously the end-effector position and the spacecraft attitude are variables cared about. In order to describe the problem, let $\mathbf{y}=\left[\begin{array}{ll}\bar{\lambda}^{T} & \mathbf{r}_{\text {end }}^{T}\end{array}\right]^{T} \in \mathfrak{R}^{6}$ be composed of attitude vector $\bar{\lambda}$ and position vector $\mathbf{r}_{\text {end }}$. Differentiating $\mathbf{y}$ with respect to time obtains

$$
\dot{\mathbf{y}}=\mathbf{J}_{y} \cdot \dot{\mathbf{q}}
$$

with

$$
\mathbf{J}_{y}=\left[\begin{array}{c}
\frac{1}{2} \boldsymbol{\Lambda}^{T} \boldsymbol{\Gamma} \\
\mathbf{J}_{L}
\end{array}\right] \in \mathfrak{R}^{6 \times N}
$$

Substituting (2) and (5) into the derivative of (6) yields the equations of motion in task space

$$
\ddot{\mathbf{y}}=\overline{\mathbf{H}}(\bar{\lambda}, \mathbf{q}) \cdot \mathbf{u}+\overline{\mathbf{C}}(\bar{\lambda}, \dot{\bar{\lambda}}, \mathbf{q}, \dot{\mathbf{q}})+\mathbf{d}(t),
$$

where $\overline{\mathbf{H}}=\mathbf{J}_{y} \mathbf{H}^{-1} \in \mathfrak{R}^{6 \times N}, \overline{\mathbf{C}}=\left[\begin{array}{c}(1 / 2) \boldsymbol{\Lambda}^{T} \dot{\Gamma} \cdot \dot{\mathbf{q}}-(1 / 4) \dot{\mathbf{q}}^{T} \boldsymbol{\Gamma}^{T} \mathbf{\Gamma} \dot{\mathbf{q}} \bar{\lambda} \\ \dot{\mathbf{J}}_{L} \dot{\mathbf{q}}\end{array}\right]-$ $\overline{\mathbf{H} C} \in \mathfrak{R}^{6 \times 6}, \mathbf{u}=\boldsymbol{\tau} \in \mathfrak{R}^{N}$ denotes the system input torque vector, and $\mathbf{d}(t)$ represents external disturbances. It is assumed that $\mathbf{d}(t)$ is bounded as $\max _{1 \leq i \leq 6}\left|d_{i}(t)\right|=\bar{d}$.

Equation (8) describes the problem in task space which is used to develop the controller.

\section{Zero-Disturbance Control Scheme}

In this section, a strategy for motion control of the spacecraft and the manipulator is presented based on integral-type sliding mode controller. Since the spacecraft actuators are off during manipulation operations, the control target is to manipulate the end-effector tracking desired trajectories by driving manipulator joints, while adjusting the spacecraft attitude by using dynamic coupling property of the system. It is possible to fulfill the above two tasks simultaneously if and only if the manipulator has kinematic redundancy with respect to the tasks [22]. Therefore, $N$ is assumed to be equal to the dimension of $\mathbf{y}$; that is, $N=6$. 
3.1. Integral-Type Sliding Mode Control. Define system tracking errors as $\mathbf{E}=\mathbf{y}-\mathbf{y}_{d}$, where $\mathbf{y}_{d}=\left[\left(\bar{\lambda}^{d}\right)^{T}\left(\mathbf{r}_{\text {end }}^{d}\right)^{T}\right]^{T} \epsilon$ $\mathfrak{R}^{6}$ represents the desired output vector, while $\mathbf{E}=$ $\left[e_{1}, e_{2}, \ldots, e_{N}\right]^{T}$.

An integral-type sliding manifold [23] is designed as follows:

$$
\mathbf{S}=\dot{\mathbf{E}}+\mathbf{K}_{1} \mathbf{E}+\mathbf{K}_{2} \int_{0}^{t} \mathbf{E} d \zeta
$$

with

$$
\begin{aligned}
& \mathbf{S}=\left[\begin{array}{llll}
s_{1} & s_{2} & \cdots & s_{N}
\end{array}\right]^{T}, \\
& \mathbf{K}_{1}=\operatorname{diag}\left(k_{11}, k_{12}, \ldots, k_{1 N}\right), \quad k_{1 i}>0 \text {, } \\
& \mathbf{K}_{2}=\operatorname{diag}\left(k_{21}, k_{22}, \ldots, k_{2 N}\right), \quad k_{2 i}>0 .
\end{aligned}
$$

Differentiating (9) with respect to time obtains

$$
\begin{aligned}
\dot{\mathbf{S}} & =\ddot{\mathbf{E}}+\mathbf{K}_{1} \dot{\mathbf{E}}+\mathbf{K}_{2} \mathbf{E} \\
& =\overline{\mathbf{H}} \cdot \mathbf{u}+\overline{\mathbf{C}}+\mathbf{K}_{1} \dot{\mathbf{E}}+\mathbf{K}_{2} \mathbf{E}-\ddot{\mathbf{y}}_{d}+\mathbf{d}(t) .
\end{aligned}
$$

From (11), the system control law is given by

$$
\begin{aligned}
\mathbf{u} & =\mathbf{u}_{\mathrm{eq}}+\mathbf{u}_{s}, \\
\mathbf{u}_{\mathrm{eq}} & =-\overline{\mathbf{H}}^{-1}\left(\overline{\mathbf{C}}+\mathbf{K}_{1} \dot{\mathbf{E}}+\mathbf{K}_{2} \mathbf{E}-\ddot{\mathbf{y}}_{d}\right), \\
\mathbf{u}_{s} & =-\overline{\mathbf{H}}^{-1} \mathbf{\Upsilon} \operatorname{sgn}(\mathbf{S}),
\end{aligned}
$$

where $\Upsilon=\operatorname{diag}\left(\gamma_{1}, \gamma_{2}, \ldots, \gamma_{N}\right) \gamma_{i}>\bar{d}$ is the switching gain matrix. In order to avoid the nonexistence of inverse of the matrix $\overline{\mathbf{H}}$, we define [5]

$$
\overline{\mathbf{H}}^{-1}= \begin{cases}\overline{\mathbf{H}}^{-1} & |\overline{\mathbf{H}}| \geq \varepsilon \\ \left(\overline{\mathbf{H}}^{T} \overline{\mathbf{H}}+\kappa^{2} \mathbf{I}\right)^{-1} \overline{\mathbf{H}}^{T} & 0<\kappa<1,|\overline{\mathbf{H}}|<\varepsilon,\end{cases}
$$

where $\varepsilon$ is a small positive constant and $\mathbf{I}$ is an identity matrix with corresponding dimension.

Theorem 1. For the dynamic system equation (8), if the integral-type sliding manifold is chosen as (9), the control law is designed as (12), then the system tracking errors will converge to zero in finite time.

Proof. Consider the following Lyapunov function candidate:

$$
\mathbf{V}_{1}=\frac{1}{2} \mathbf{S}^{T} \mathbf{S}
$$

Substituting (12) into the first derivative of (14) yields

$$
\begin{aligned}
\dot{\mathbf{V}}_{1} & =\mathbf{S}^{T}\left[\overline{\mathbf{H}}\left(\mathbf{u}_{\mathrm{eq}}+\mathbf{u}_{s}\right)+\overline{\mathbf{C}}+\mathbf{K}_{1} \dot{\mathbf{E}}+\mathbf{K}_{2} \mathbf{E}-\ddot{\mathbf{y}}_{d}+\mathbf{d}(t)\right] \\
& =\mathbf{S}^{T}[\mathbf{d}(t)-\mathbf{Y} \operatorname{sgn}(\mathbf{S})] \\
& \leq \sum_{i=1}^{N}\left(\bar{d}-\gamma_{i}\right)\left|s_{i}\right| .
\end{aligned}
$$

Obviously, the sliding condition $\mathbf{S}^{T} \dot{\mathbf{S}}<0$ is satisfied; that is, $\dot{\mathbf{V}}_{1}<0$ for $\forall s_{i} \neq 0$. Hence, the closed-loop control system is globally stable by Lyapunov stability criterion.
3.2. Design of Adaptive Switching Gains. In practice the unknown disturbances are difficult to estimate. In order to guarantee high robustness, large switching gains must be set for the controller, which induce serious chattering. Therefore, in this subsection the adaptive laws are adopted to update the switching gains online, so as to suppress the chattering problem and improve the control precision.

Assuming that $\Upsilon^{*}$ denotes the ideal switching gain and $\widehat{\Upsilon}$ is the estimated value of $\mathbf{Y}^{*}, \mathbf{u}_{s}$ can be rewritten as

$$
\mathbf{u}_{s}=-\overline{\mathbf{H}}^{-1} \widehat{\mathbf{Y}} \operatorname{sgn}(\mathbf{S}) .
$$

Theorem 2. For the dynamic system equation (8), if (9) is chosen as the sliding manifold, the control law is defined as (12) and (16), then the system tracking errors will converge to zero in finite time, while the adaptive law is designed as follows:

$$
\dot{\hat{\mathbf{Y}}}=\mathbf{A} \cdot a b s(\mathbf{S})
$$

with $\mathbf{A}=\operatorname{diag}\left(\alpha_{1}, \ldots, \alpha_{N}\right), \alpha_{i}>0, a b s(\mathbf{S})=$

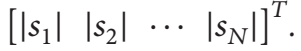

Proof. Consider the Lyapunov function candidate as

$$
\mathbf{V}_{2}=\frac{1}{2} \mathbf{S}^{T} \mathbf{S}+\frac{1}{2} \widetilde{\mathbf{Y}}^{T} \mathbf{A}^{-1} \widetilde{\mathbf{Y}}
$$

where $\widetilde{\Upsilon}=\widehat{\Upsilon}-\Upsilon^{*}$ is the estimation error and holds for $\dot{\widetilde{\Upsilon}}=\dot{\widehat{\Upsilon}}$.

Substituting (12)-(17) into the first derivative of (18) results in

$$
\begin{aligned}
\dot{\mathbf{V}}_{2} & =\mathbf{S}^{T}[\mathbf{d}(t)-\widehat{\mathbf{Y}} \operatorname{sgn}(\mathbf{S})]+\widetilde{\mathbf{\Upsilon}}^{T} \mathbf{A}^{-1} \dot{\hat{\mathbf{Y}}} \\
& =\mathbf{S}^{T}\left[\mathbf{d}(t)-\mathbf{\Upsilon}^{*} \operatorname{sgn}(\mathbf{S})\right]+\widetilde{\mathbf{\Upsilon}}^{T} \mathbf{A}^{-1}[\dot{\hat{\mathbf{Y}}}-\mathbf{A} \cdot \operatorname{abs}(\mathbf{S})] \\
& \leq-\sum_{i=1}^{N}\left(\gamma_{i}^{*}-d_{i}\right)\left|s_{i}\right| \leq 0 .
\end{aligned}
$$

From (19), the sliding condition is satisfied. Therefore, the system control errors will converge to zero in finite time.

\section{Simulation Results}

Simulations are carried out on a free-floating planar system with three-link rigid manipulator shown as Figure 1. The physical parameters of the system are shown in Table 1. In planar case, the spacecraft attitude can be simplified as angle $\varphi$ along $z$ axis. The external disturbances are given by $\mathbf{d}(t)=$ $0.2 \times[3 \sin (\pi t / 5)+\cos (\pi t / 5)]$.

In this section, the manipulator is controlled to track a desired trajectory in task space while the spacecraft attitude remains undisturbed. In order to avoid dynamic singularities, the desired trajectory is chosen in the Path Independent Workspace [24]. The desired trajectory is defined as

$$
\begin{aligned}
& x_{e}^{d}=2+0.2 \cdot \cos \left(\frac{\pi t}{10}\right), \\
& y_{e}^{d}=1+0.2 \cdot \sin \left(\frac{\pi t}{10}\right) .
\end{aligned}
$$


TABLE 1: Physical parameters of the planar system.

\begin{tabular}{lcccc}
\hline Body & $r_{i} / \mathrm{m}$ & $l_{i} / \mathrm{m}$ & $m_{i} / \mathrm{kg}$ & $I_{i} /\left(\mathrm{kg} \cdot \mathrm{m}^{2}\right)$ \\
\hline 0 & 0.5 & 0.5 & 40 & 6.667 \\
1 & 0.5 & 0.5 & 4 & 0.333 \\
2 & 0.5 & 0.5 & 3 & 0.250 \\
3 & 0.5 & 0.5 & 3 & 0.250 \\
\hline
\end{tabular}

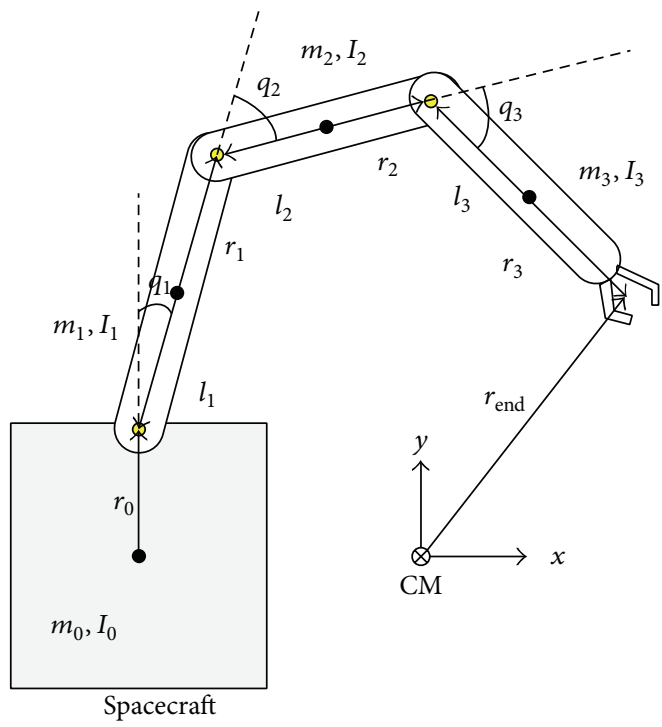

FIGURE 1: A planar space manipulator system with three-link manipulator.

Given the initial attitude of the system as $\varphi(0)=0, q_{1}(0)=$ $30^{\circ}, q_{2}(0)=-45^{\circ}, q_{3}(0)=36^{\circ}$, the spacecraft attitude is expected to remain at $\varphi(t)=0$. The proposed adaptive integral-type sliding mode controller (AISMC) is applied to such planar system with parameters being designed as follows:

$$
\begin{aligned}
& \mathbf{K}_{1}=\operatorname{diag}(800,800,800), \\
& \mathbf{K}_{2}=\operatorname{diag}(400,400,400), \\
& \mathbf{A}=\operatorname{diag}(0.01,0.01,0.01) .
\end{aligned}
$$

For the purpose of comparison, the integral-type sliding mode controller (ISMC) without adaptive gains is also applied to the same system. The corresponding parameters are the same as the above. Finally, the simulation results are shown in Figures 2-6.

Figure 2 shows trajectories of the manipulator end in the task space, where the red line is the desired path and the black lines are the actual trajectories corresponding to the controllers with and without adaptive gains. It can be seen that the manipulator end moves along the desired trajectory smoothly. But, from the zoomed image on the upper right of Figure 2, the AISMC performs better than the ISMC. The spacecraft attitude during the manipulation is shown in Figure 3. It is seen that the spacecraft attitude varies in the scale of $10^{-3}$ degrees under the ISMC, while it is relatively

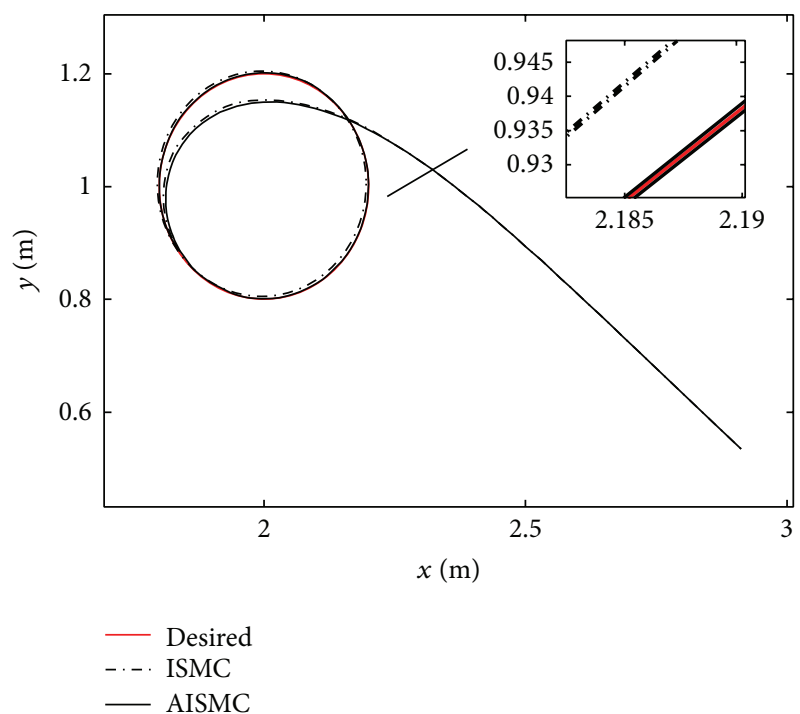

FIgURE 2: The manipulator end trajectories.

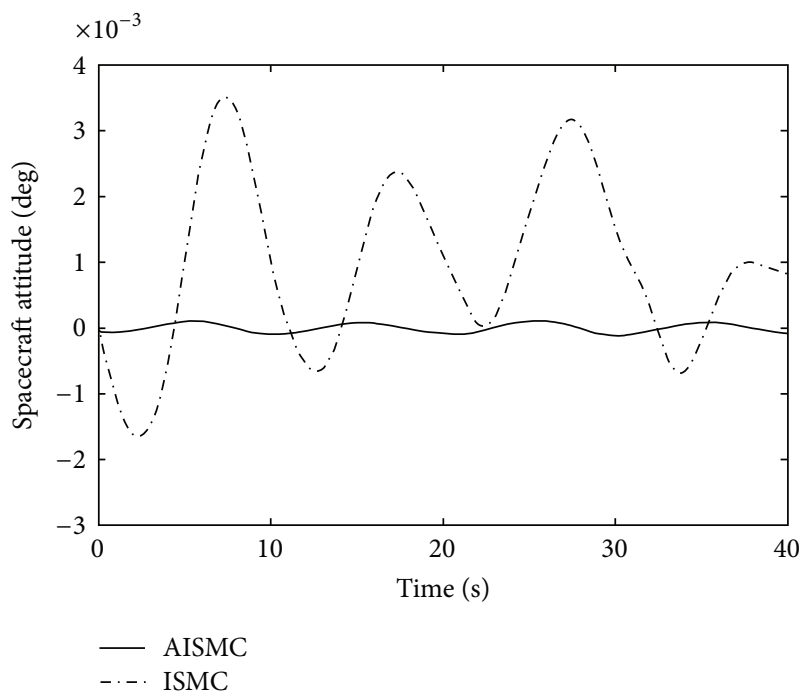

FIgURE 3: The spacecraft attitude trajectories.

smaller when the AISMC is used. It may be concluded that the attitude of the spacecraft is almost undisturbed.

Figure 4 shows the tracking errors of $x$ - and $y$ coordinates in the task space. It is clear that the tracking errors converge to zero over time and the AISMC converges more precisely than the ISMC. In Figure 5 the trajectories of manipulator joints are presented. These trajectories are smooth throughout the motion in Figure 2.

Figure 6 (left) shows control torques acting on the manipulator joints, which are required for the motion in Figure 2. Figure 6 (right) is a magnified image of the rectangular area of the left figure. It can be seen that the adaptive gains effectively reduce chattering in practice. 


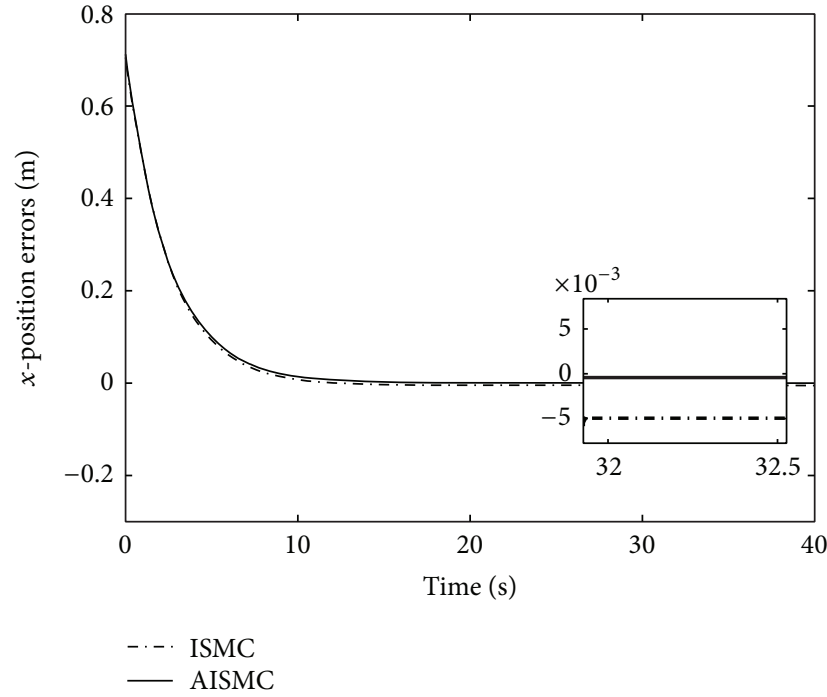

(a) $x$-position

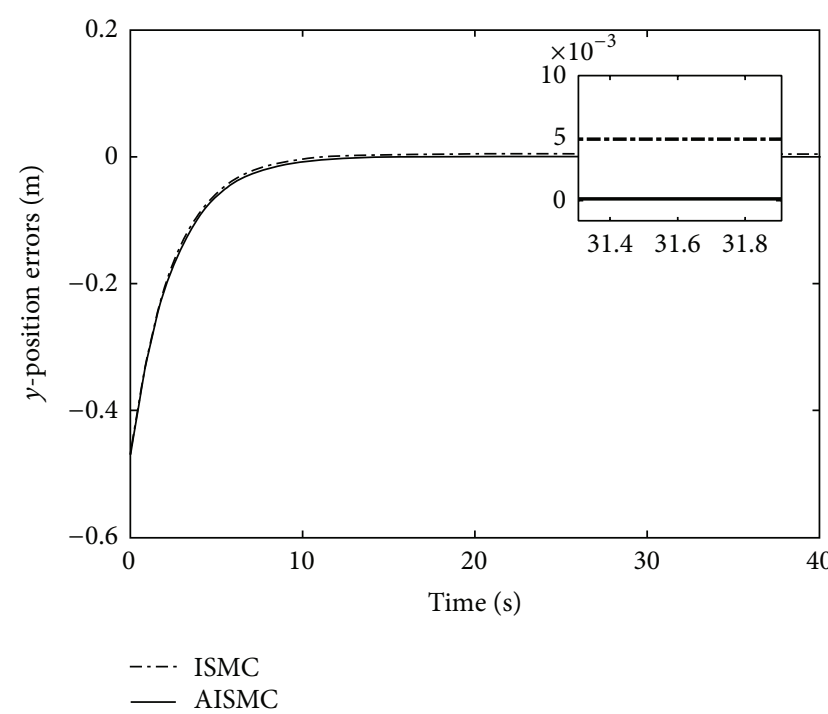

(b) $y$-position

FIgURE 4: Position tracking errors of the manipulator.

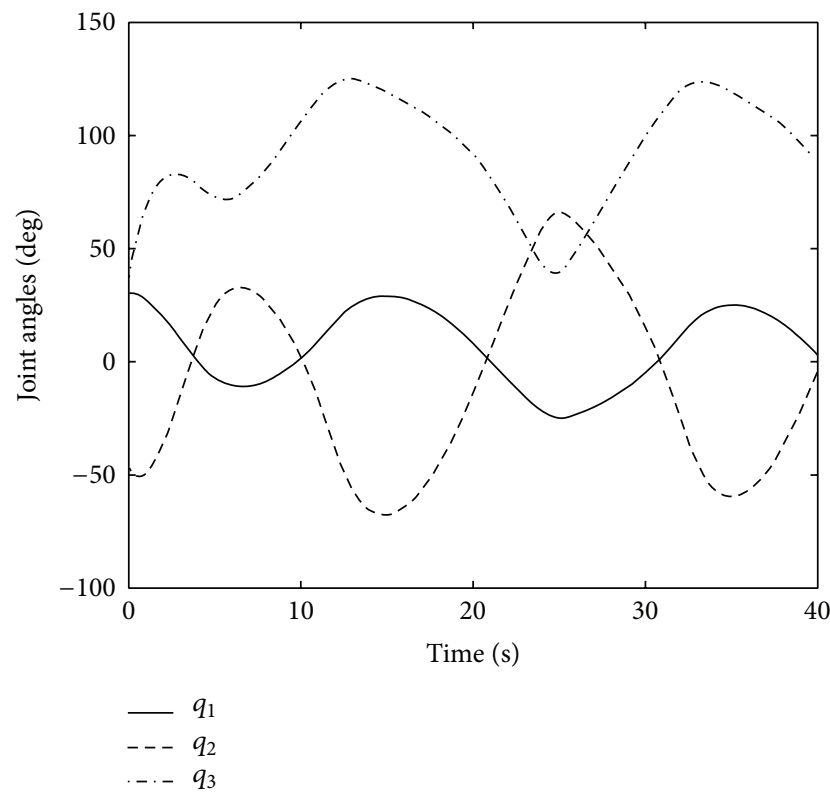

(a)

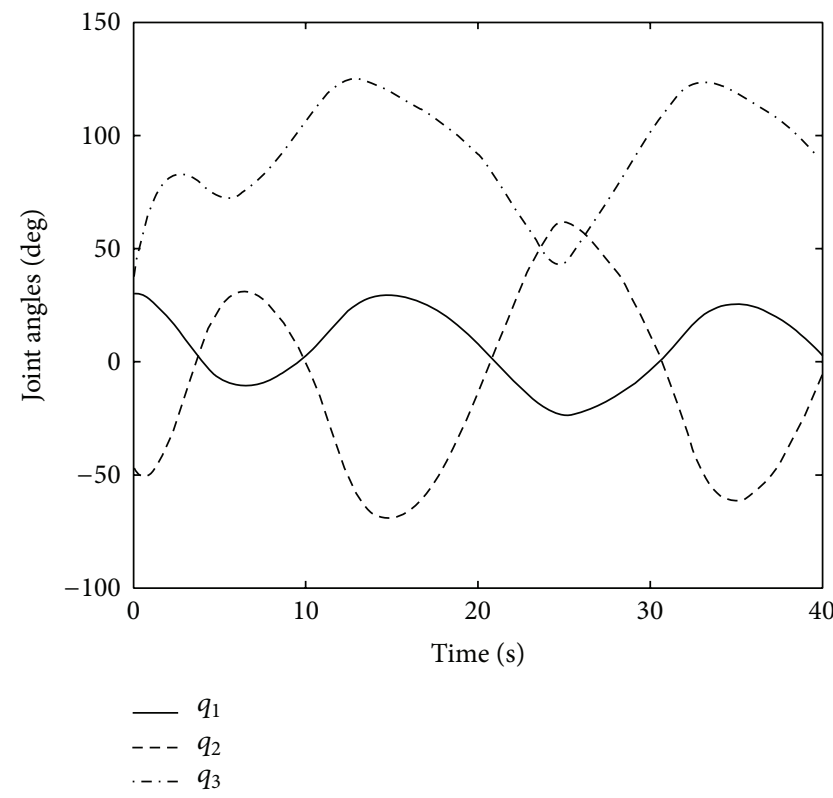

(b)

FIGURE 5: Trajectories in joint space.

\section{Conclusion}

A zero-disturbance control scheme for free-floating space manipulator systems is investigated in the paper. With only the manipulator joints being actuated, the spacecraft attitude is changeless during the manipulations, which is achieved by using dynamic coupling property of the free-floating system rather than actively controlling thrusters of the spacecraft. In contrast to traditional method, the precious fuel or electric power is saved. The application of the method is illustrated using a $2 \mathrm{D}$ example. The simulation results show that the manipulator joints are actuated smoothly to move endeffector along the desired trajectories, while the spacecraft attitude is undisturbed. Overall, the results demonstrate the good performance of the proposed method. It has broad application prospect in on-orbit manipulations. 

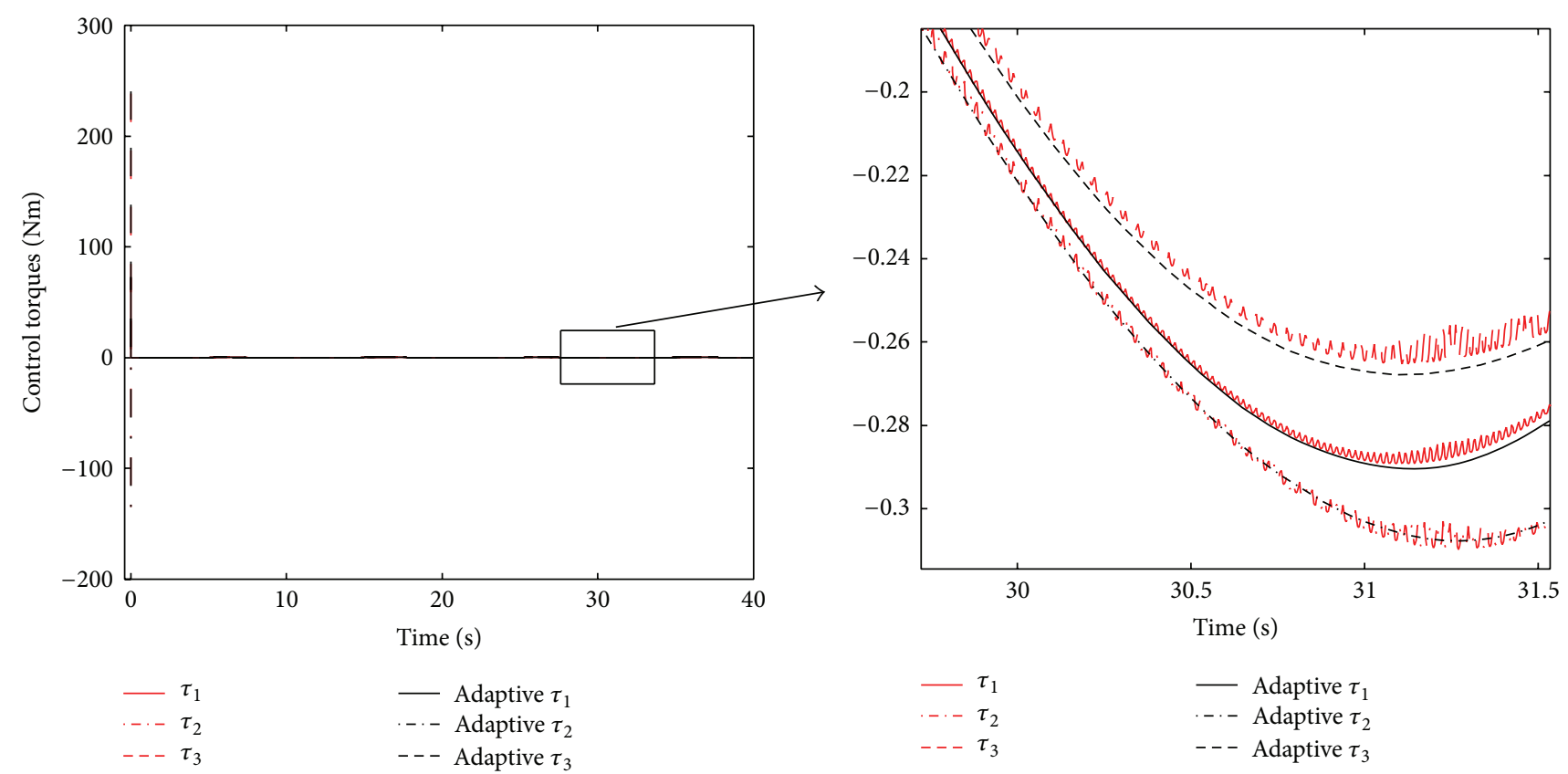

FIGURE 6: Control torques acting on the manipulator joints.

\section{Appendix}

Equation (4) is rewritten here as

$$
\dot{\lambda}=\left[\begin{array}{c}
\dot{\lambda}_{0} \\
\dot{\bar{\lambda}}
\end{array}\right]=\frac{1}{2} \mathbf{G} \cdot \omega_{0},
$$

where $\mathbf{G}=\left[\begin{array}{ll}-\bar{\lambda} & \boldsymbol{\Lambda}\end{array}\right]^{T} \in \mathfrak{R}^{4 \times 3}$ and it is easy to find

$$
\begin{gathered}
\mathbf{G}^{T} \mathbf{G}=\mathbf{I}_{3}, \\
\mathbf{G G}^{T}=\mathbf{I}_{4}-\lambda \boldsymbol{\lambda}^{T} .
\end{gathered}
$$

Using (A.1) and (A.2), it is obtained that

$$
\omega_{0}=2 \mathbf{G}^{T} \dot{\lambda}
$$

Differentiating (A.1) with respect to time yields

$$
\ddot{\lambda}=\frac{1}{2} \dot{\mathbf{G}} \boldsymbol{\omega}_{0}+\frac{1}{2} \mathbf{G} \dot{\omega}_{0} .
$$

Multiplying both sides of (A.3) by $\dot{\mathbf{G}}$ results in

$$
\dot{\mathbf{G}} \dot{\omega}_{0}=2 \dot{\mathbf{G}} \mathbf{G}^{T} \dot{\lambda} .
$$

Differentiating the lower part of (A.2) and substituting it into (A.5) yield

$$
\dot{\mathbf{G}} \dot{\boldsymbol{\omega}}_{0}=-2\left(\mathrm{G} \dot{\mathbf{G}}^{T} \dot{\lambda}+\dot{\lambda} \boldsymbol{\lambda}^{T} \dot{\lambda}+\lambda \dot{\lambda}^{T} \dot{\lambda}\right)
$$

Since $\dot{\mathbf{G}}$ and $\dot{\boldsymbol{\lambda}}$ satisfy the properties

$$
\dot{\mathbf{G}} \boldsymbol{\lambda}=0, \quad \lambda^{T} \dot{\lambda}=0, \quad 4 \dot{\lambda}^{T} \dot{\lambda}=\boldsymbol{\omega}_{0}^{T} \boldsymbol{\omega}_{0},
$$

putting (A.7) into (A.6) obtains

$$
\dot{\mathrm{G}} \dot{\omega}_{0}=-\frac{1}{2} \omega_{0}^{T} \omega_{0} \lambda
$$

Using (A.8), (A.4) is rewritten as

$$
\ddot{\lambda}=\frac{1}{2} \mathrm{G} \dot{\omega}_{0}-\frac{1}{4} \omega_{0}^{T} \omega_{0} \lambda .
$$

Substituting (3) and its derivative into (A.9) results in

$$
\ddot{\lambda}=\frac{1}{2} \mathbf{G}(\dot{\Gamma} \dot{\mathbf{q}}+\Gamma \ddot{\mathbf{q}})-\frac{1}{4} \dot{\mathbf{q}}^{T} \boldsymbol{\Gamma}^{T} \boldsymbol{\Gamma} \dot{\mathbf{q}} \lambda,
$$

which is equal to (5).

\section{Conflict of Interests}

The authors declare that there is no conflict of interests regarding the publication of this paper.

\section{Acknowledgments}

This work was supported by the Hunan Province Scientific and Technological Research Projects (2013SK4030) and Education Department of Hunan Province (14C0952).

\section{References}

[1] R. Rembala and C. Ower, "Robotic assembly and maintenance of future space stations based on the ISS mission operations experience," Acta Astronautica, vol. 65, no. 7-8, pp. 912-920, 2009.

[2] K. Yoshida, "Achievements in space robotics," IEEE Robotics and Automation Magazine, vol. 16, no. 4, pp. 20-28, 2009. 
[3] K. Nanos and E. Papadopoulos, "On the use of free-floating space robots in the presence of angular momentum," Intelligent Service Robotics, vol. 4, no. 1, pp. 3-15, 2011.

[4] W. Xu, C. Li, B. Liang, Y. Xu, Y. Liu, and W. Qiang, "Target berthing and base reorientation of free-floating space robotic system after capturing," Acta Astronautica, vol. 64, no. 2-3, pp. 109-126, 2009.

[5] W. Xu, B. Liang, and Y. Xu, "Practical approaches to handle the singularities of a wrist-partitioned space manipulator," Acta Astronautica, vol. 68, no. 1-2, pp. 269-300, 2011.

[6] P. J. From, K. Y. Pettersen, and J. T. Gravdahl, "Singularity-free dynamic equations of spacecraft-manipulator systems," Acta Astronautica, vol. 69, no. 11-12, pp. 1057-1065, 2011.

[7] W. Xu, B. Liang, and Y. Xu, "Survey of modeling, planning, and ground verification of space robotic systems," Acta Astronautica, vol. 68, no. 11-12, pp. 1629-1649, 2011.

[8] S. A. A. Moosavian and E. Papadopoulos, "Free-flying robots in space: an overview of dynamics modeling, planning and control," Robotica, vol. 25, no. 5, pp. 537-547, 2007.

[9] S. Dubowsky and E. Papadopoulos, "The kinematics, dynamics, and control of free-flying and free-floating space robotic systems," IEEE Transactions on Robotics and Automation, vol. 9, no. 5, pp. 531-543, 1993.

[10] W. Xu, Y. Liu, B. Liang, Y. Xu, C. Li, and W. Qiang, "Nonholonomic path planning of a free-floating space robotic system using genetic algorithms," Advanced Robotics, vol. 22, no. 4, pp. 451-476, 2008.

[11] E. Papadopoulos, I. Tortopidis, and K. Nanos, "Smooth planning for free-floating space robots using polynomials," in Proceedings of the IEEE International Conference on Robotics and Automation, pp. 4272-4277, Barcelona, Spain, April 2005.

[12] W. F. Xu, X. Q. Wang, Q. Xue, and B. Liang, "Study on trajectory planning of dual-arm space robot keeping the base stabilized," Acta Automatica Sinica, vol. 39, no. 1, pp. 69-80, 2013.

[13] K. Yoshida, "Engineering test satellite VII flight experiments for space robot dynamics and control: theories on laboratory test beds ten years ago, now in orbit," The International Journal of Robotics Research, vol. 22, no. 5, pp. 321-335, 2003.

[14] D. N. Nenchev, "Reaction null space of a multibody system with applications in robotics," Mechanical Sciences, vol. 4, no. 1, pp. 97-112, 2013.

[15] Y. Liao, D. Li, and G. Tang, "Translational zero-disturbance curve and its application to zero-disturbance motion planning of space manipulator system," Science China Technological Sciences, vol. 54, no. 5, pp. 1234-1239, 2011.

[16] O. Parlaktuna and M. Ozkan, "Adaptive control of free-floating space manipulators using dynamically equivalent manipulator model," Robotics and Autonomous Systems, vol. 46, no. 3, pp. 185-193, 2004.

[17] S. A. A. Moosavian and E. Papadopoulos, "Control of space free-flyers using the modified transpose Jacobian algorithm," in Proceedings of the IEEE/RSJ International Conference on Intelligent Robots and Systems (IROS '97), vol. 3, pp. 1500-1505, IEEE, Grenoble, France, September 1997.

[18] H. Khaloozadeh and M. R. Homaeinejad, "Real-time regulated sliding mode controller design of multiple manipulator space free-flying robot," Journal of Mechanical Science and Technology, vol. 24, no. 6, pp. 1337-1351, 2010.

[19] Y. Guo and L. Chen, "Adaptive sliding mode control for coordinated motion of free-floating space manipulators based on filtering technique," in Proceedings of the ASME International
Design Engineering Technical Conferences and Computers and Information in Engineering Conference (DETC '09), pp. 17931798, San Diego, Calif, USA, September 2009.

[20] F. Zhang, Y. Fu, L. Hua, H. Chen, S. Wang, and B. Guo, "Pointto-point planning for free-floating space manipulator with zerodisturbance spacecraft attitude," in Proceedings of the IEEE International Conference on Information and Automation (ICIA '12), pp. 142-147, Shenyang, China, June 2012.

[21] F. Aghili, "Coordination control of a free-flying manipulator and its base attitude to capture and detumble a noncooperative satellite," in Proceedings of the IEEE/RSJ International Conference on Intelligent Robots and Systems (IROS '09), pp. 2365-2372, St. Louis, Mo, USA, October 2009.

[22] D. N. Nenchev, K. Yoshida, P. Vichitkulsawat, and M. Uchiyama, "Reaction null-space control of flexible structure mounted manipulator systems," IEEE Transactions on Robotics and Automation, vol. 15, no. 6, pp. 1011-1023, 1999.

[23] Q. Hu, Y. Zhang, X. Huo, and B. Xiao, "Adaptive integral-type sliding mode control for spacecraft attitude maneuvering under actuator stuck failures," Chinese Journal of Aeronautics, vol. 24, no. 1, pp. 32-45, 2011

[24] K. Nanos and E. Papadopoulos, "On Cartesian motions with singularities avoidance for free-floating space robots," in Proceedings of the IEEE International Conference on Robotics and Automation (ICRA '12), pp. 5398-5403, St Paul, Minn, USA, May 2012. 


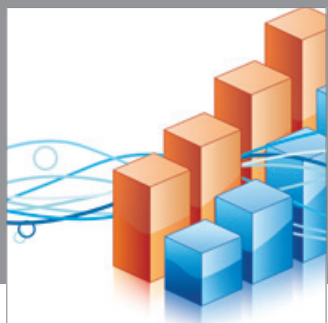

Advances in

Operations Research

mansans

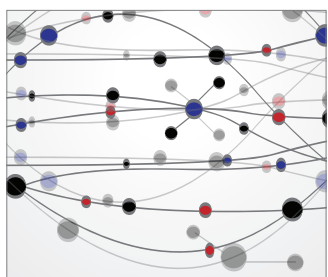

The Scientific World Journal
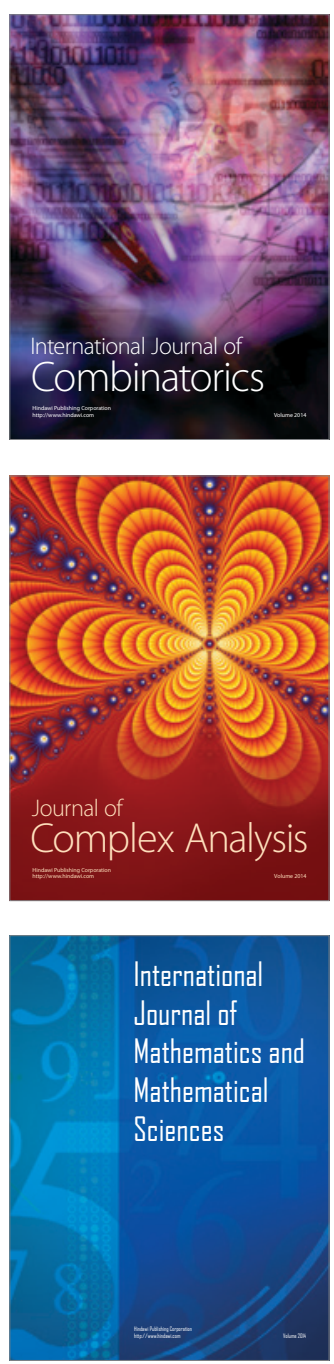
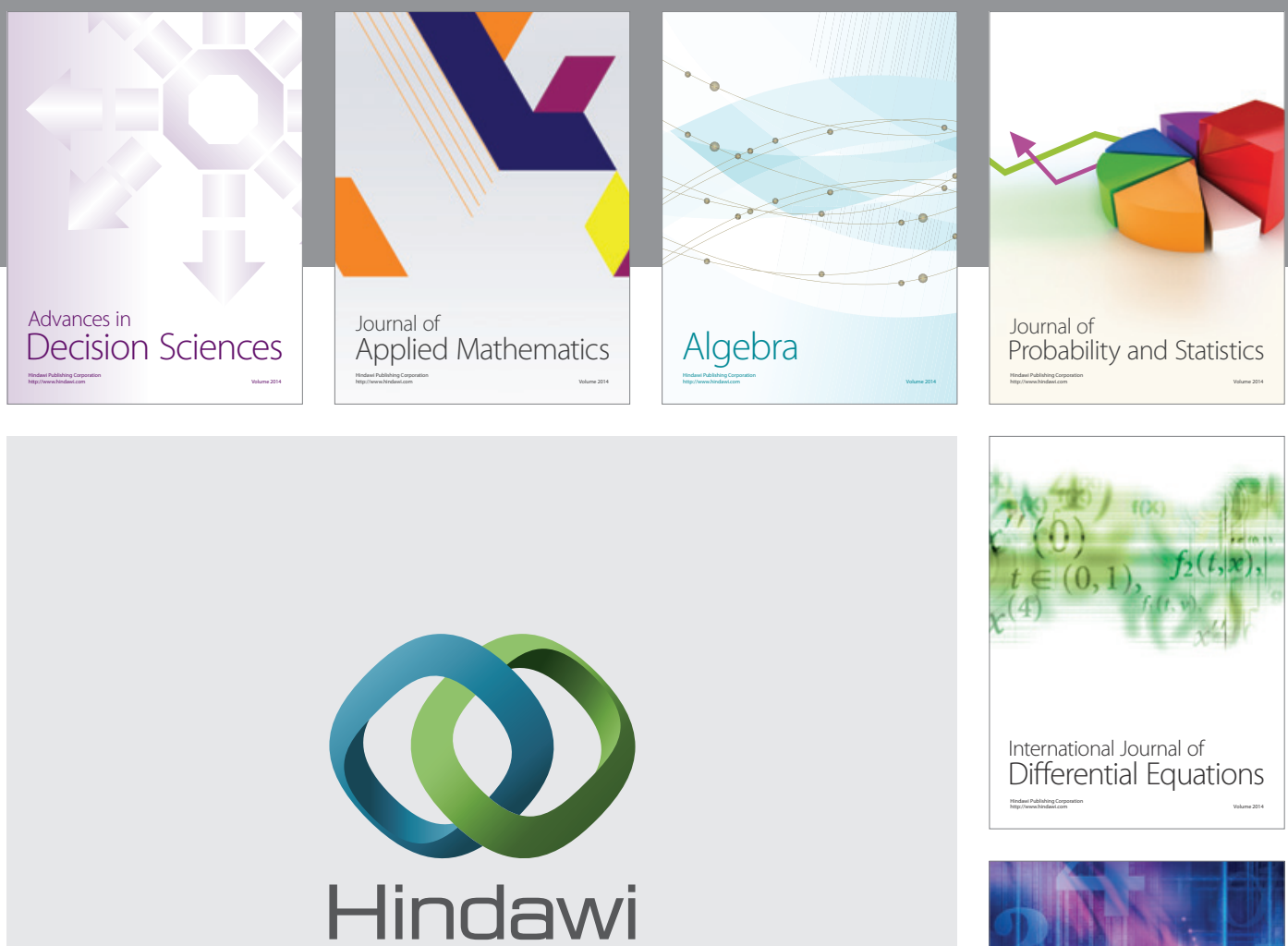

Submit your manuscripts at http://www.hindawi.com
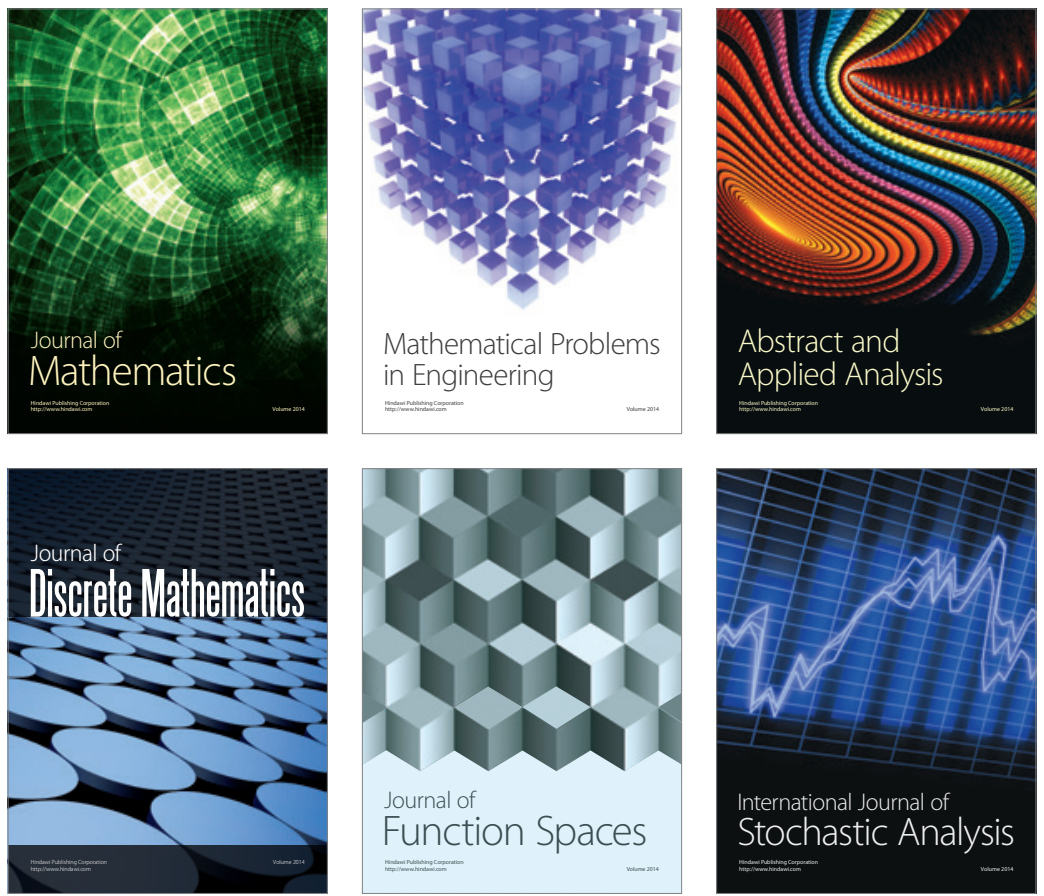

Journal of

Function Spaces

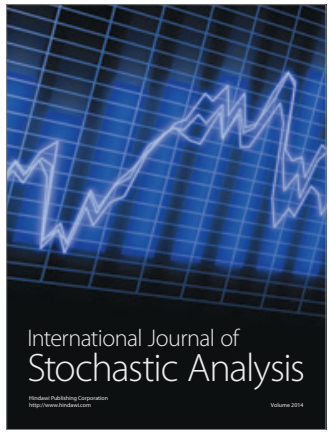

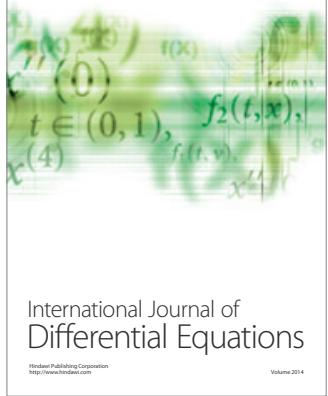
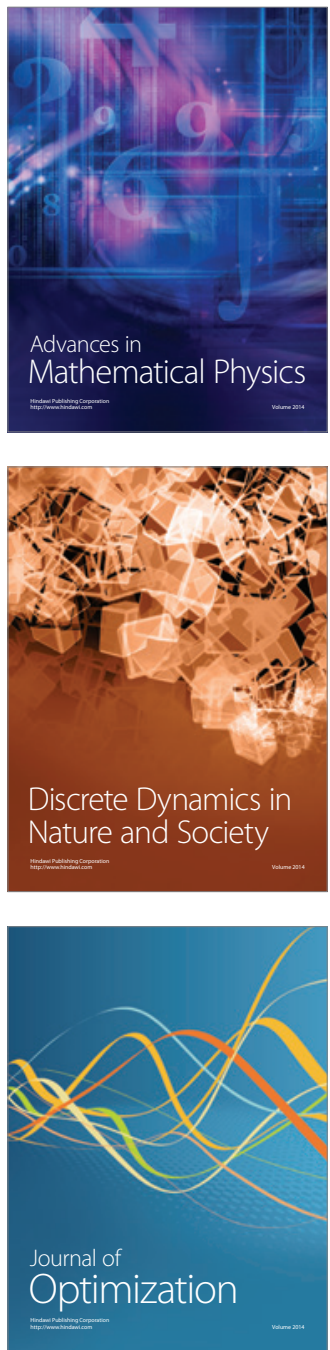